Internat. J. Math. \& Math. Sci.

Vol. 24, No. 9 (2000) 613-616

S0161171200004002

(C) Hindawi Publishing Corp.

\title{
BIORTHOGONALITY CONDITION FOR CREEPING MOTION IN ANNULAR TRENCHES
}

\author{
SUHEIL A. KHURI
}

(Received 15 October 1999)

\begin{abstract}
The biorthogonality condition for Stokes flow in annular trenches bounded by horizontal parallel planes and concentric vertical cylinders is derived. This condition, is needed to compute the coefficients of the eigenfunction expansion solution of the corresponding Stokes flow problem.
\end{abstract}

Keywords and phrases. Eigenvalues, eigenfunctions, eigenfunction expansion, biorthogonality conditions, Stokes flow.

2000 Mathematics Subject Classification. Primary 76D07, 76D99, 35P10.

1. Introduction. Recently, the eigenfunction expansion method has been used extensively for solving problems of Stokes flow; (cf. [1, 2, 7, 8]). Most recently, biorthogonality conditions were used by Khuri to solve Stokes flow in a sectorial cavity [3] and by Khuri and Wang for solving Stokes flow around a bend [6]. The method leads to the development of a set of eigenfunctions, adjoint eigenfunctions, biorthogonality conditions and an algorithm for the computation of the eigenfunction expansions.

In the present paper, we derive the biorthogonality condition for Stokes flow in annular trenches bounded by horizontal parallel planes and concentric vertical cylinders. For biorthogonality conditions in other flow geometries, derived in a similar fashion, see $[4,5]$.

2. Biorthogonality condition. In this section, we state Theorem 2.1 which gives the biorthogonality condition satisfied by the eigenfunctions and adjoint eigenfunctions of the following fourth-order boundary value problem:

$$
\left(P_{0}(r) y^{\prime \prime}(r)\right)^{\prime \prime}+\left(P_{1}(r ; \alpha) y^{\prime}(r)\right)^{\prime}+P_{2}(r ; \alpha) y(r)=0, \quad r \in\left[r_{1}, r_{2}\right] .
$$

The boundary conditions are given by

$$
y\left(r_{1}\right)=y\left(r_{2}\right)=y^{\prime}\left(r_{1}\right)=y^{\prime}\left(r_{2}\right)=0 .
$$

This biorthogonality condition was proved by Khuri [3].

THEOREM 2.1 (biorthogonality condition). Consider the boundary value problem given in (2.1) and (2.2) where $P_{0}(r), P_{1}^{\prime \prime}(r ; \alpha), P_{2}(r ; \alpha)$ are continuous and $P_{0}(r) \neq 0$ on $r_{1} \leq r \leq r_{2} . P_{i}$ in (2.1) is a polynomial of degree at most $i$ in the parameter $\alpha$, where $i=0,1,2$, in particular, let $P_{1}(r ; \alpha)=p_{11}(r) \alpha+p_{12}(r)$, and we require

$$
P_{1}^{2}(r ; \alpha)-4 P_{0}(r) P_{2}(r ; \alpha)=p_{31}(r) \alpha+p_{32}(r), \quad p_{11}^{2}(r)+p_{31}^{2}(r) \not \equiv 0 .
$$


Then with $P_{n}^{*}$ defined by

$$
P_{n}^{*}=\int_{r_{1}}^{r_{2}}\left[\phi_{2}^{(n)}(r), \phi_{1}^{(n)}(r)\right] B(r)\left[\begin{array}{l}
\phi_{1}^{(n)}(r) \\
\phi_{2}^{(n)}(r)
\end{array}\right] d r,
$$

we have the following biorthogonality condition:

$$
\int_{r_{1}}^{r_{2}}\left[\phi_{2}^{(m)}(r), \phi_{1}^{(m)}(r)\right] B(r)\left[\begin{array}{l}
\phi_{1}^{(n)}(r) \\
\phi_{2}^{(n)}(r)
\end{array}\right] d r=P_{n}^{*} \delta_{m n},
$$

where $\delta_{m n}$ is the Kronecker's delta,

$$
B(r)=\left(\begin{array}{cc}
-\frac{1}{2} \frac{p_{11}(r)}{P_{0}(r)} & 0 \\
\frac{1}{2} p_{11}^{\prime \prime}(r)+\frac{1}{4} \frac{p_{31}(r)}{P_{0}(r)} & -\frac{1}{2} \frac{p_{11}(r)}{P_{0}(r)}
\end{array}\right)
$$

with

$$
\begin{gathered}
\phi_{1}^{(n)}(r)=y_{n}(r), \\
\phi_{2}^{(n)}(r)=P_{0}(r) y_{n}^{\prime \prime}(r)+\frac{1}{2} P_{1}\left(r ; \alpha_{n}\right) y_{n}(r) .
\end{gathered}
$$

Here $y_{i}$ is an eigenfunction of (2.1) corresponding to the eigenvalue $\alpha_{i}$. Assume the eigenvalues $\alpha_{i}$ are simple.

3. Stokes flow in annular trenches. Next, we derive the biorthogonality condition for Stokes flow in annular trenches bounded by horizontal parallel planes and concentric vertical cylinders which was studied by Yoo and Joseph [8]. The region is given by

$$
v=\left\{r, z: 0<r_{1} \leq r \leq r_{2},-z_{1} \leq z \leq z_{1}\right\} .
$$

The Stokes flow equation in $v$ for axisymmetric flow in cylindrical coordinates is

$$
E^{4} \Psi(r, z)=\left(\frac{\partial^{2}}{\partial r^{2}}-\frac{1}{r} \frac{\partial}{\partial r}+\frac{\partial^{2}}{\partial z^{2}}\right)^{2} \Psi(r, z)=0 .
$$

We require the velocity to vanish on the rigid boundaries of the two concentric cylinders where $r=r_{1}, r_{2}$ so we need

$$
\Psi\left(r_{1}, z\right)=\Psi\left(r_{2}, z\right)=\frac{\partial \Psi}{\partial r}\left(r_{1}, z\right)=\frac{\partial \Psi}{\partial r}\left(r_{2}, z\right)=0 .
$$

Other edge data are prescribed on $z= \pm z_{1}$ as in [8].

Separable solutions of (3.2) and (3.3) in the form

$$
\Psi(r, z) \sim e^{ \pm p z} T(r)
$$

exist [8], when $T(r)$ satisfies the following equation:

$$
T^{(4)}-\frac{2}{r} T^{(3)}+\left(2 p^{2}+\frac{3}{r^{2}}\right) T^{(2)}-\left(\frac{2 p^{2}}{r}+\frac{3}{r^{3}}\right) T^{(1)}+p^{4} T=0
$$


and the boundary conditions

$$
T\left(r_{1}\right)=T\left(r_{2}\right)=T^{\prime}\left(r_{1}\right)=T^{\prime}\left(r_{2}\right)=0 .
$$

Clearly (3.5) can be rewritten in the following form:

$$
\left(\frac{1}{r} T^{\prime \prime}\right)^{\prime \prime}+\left(\left[\frac{2 p^{2}}{r}+\frac{1}{r^{3}}\right] T^{\prime}\right)^{\prime}+\frac{p^{4}}{r} T=0 .
$$

Comparing with Theorem 2.1, we require $\alpha_{n} \neq \alpha_{m}$ and we have

$$
P_{0}(r)=\frac{1}{r}, \quad P_{1}(r ; \alpha)=\frac{2 \alpha}{r}+\frac{1}{r^{3}}, \quad P_{2}(r ; \alpha)=\frac{\alpha^{2}}{r},
$$

where

$$
\alpha=p^{2}
$$

Since

$$
P_{1}^{2}(r ; \alpha)-4 P_{0}(r) P_{2}(r ; \alpha)=\frac{4 \alpha}{r^{4}}+\frac{1}{r^{6}}
$$

thus

$$
p_{31}(r)=\frac{4}{r^{4}}, \quad p_{32}(r)=\frac{1}{r^{6}}
$$

Clearly,

$$
p_{11}(r)=\frac{2}{r} ; \quad p_{12}(r)=\frac{1}{r^{3}} .
$$

By applying Theorem 2.1, the biorthogonality condition is given by

$$
\int_{r_{1}}^{r_{2}}\left[\phi_{1}^{(m)}(r), \phi_{2}^{(m)}(r)\right] B(r)\left[\begin{array}{l}
\phi_{1}^{(n)}(r) \\
\phi_{2}^{(n)}(r)
\end{array}\right] d r=P_{n}^{*} \delta_{m n}, \quad\left(p_{n}^{2} \neq p_{m}^{2}\right),
$$

where

$$
B(r)=\left(\begin{array}{cc}
-1 & 0 \\
\frac{3}{r^{3}} & -1
\end{array}\right)
$$

The eigenfunctions satisfy

$$
\begin{gathered}
\phi_{1}^{(n)}(r)=T_{n}(r), \\
\phi_{2}^{(n)}(r)=\frac{1}{r} T_{n}^{\prime \prime}(r)+\left(\frac{1}{r} \alpha_{n}+\frac{1}{2 r^{3}}\right) T_{n}(r)
\end{gathered}
$$

and the adjoint eigenfunctions satisfy

$$
\begin{gathered}
\psi_{1}^{(m)}(r)=\frac{1}{r} T_{m}^{\prime \prime}(r)+\left(\frac{1}{r} \alpha_{m}+\frac{2}{r^{3}}\right) T_{m}(r), \\
\psi_{2}^{(m)}(r)=T_{m}(r),
\end{gathered}
$$

where

$$
\alpha_{n}=p_{n}^{2}
$$


A similar biorthogonality condition was derived by Yoo and Joseph [8], where $\phi^{(n)}$ and $\psi^{(n)}$ were defined through a two-dimensional eigenvalue problem. The eigenfunctions and adjoint eigenfunctions that we have derived are, however, given explicitly in terms of $T_{n}$.

\section{REFERENCES}

[1] D. D. Joseph and L. Sturges, The convergence of biorthogonal series for biharmonic and Stokes flow edge problems. II, SIAM J. Appl. Math. 34 (1978), no. 1, 7-26. MR 57\#14765. Zbl 379.31001.

[2] D. D. Joseph, L. D. Sturges, and W. H. Warner, Convergence of biorthogonal series of biharmonic eigenfunctions by the method of Titchmarsh, Arch. Rational Mech. Anal. 78 (1982), no. 3, 223-274. MR 84i:35108. Zbl 486.35061.

[3] S. A. Khuri, Biorthogonal series solution of Stokes flow problems in sectorial regions, SIAM J. Appl. Math. 56 (1996), no. 1, 19-39. MR 96i:76035. Zbl 844.76019.

[4] _ Biorthogonality condition for axisymmetric Stokes flow in a toroidal region, Appl. Math. Comput. 97 (1998), no. 2-3, 255-259. MR 99f:76043. Zbl 990.39993.

[5] _ Biorthogonality condition for Stokes flow in rectangular regions, Int. J. Comput. Math. 70 (1999), no. 3, 411-415. CMP 1712 658. Zbl 916.76016.

[6] S. A. Khuri and C. Y. Wang, Stokes flow around a bend, Quart. Appl. Math. 55 (1997), no. 3, 573-600. MR 98d:76042. Zbl 887.76020.

[7] C. H. Liu and D. D. Joseph, Stokes flow in wedge-shaped trenches, J. Fluid Mech. 80 (1977), 443-463.

[8] J. Y. Yoo and D. D. Joseph, Stokes flow in a trench between concentric cylinders, SIAM J. Appl. Math. 34 (1978), no. 2, 247-285. MR 80d:76033. Zbl 376.76019.

SUHEIL A. KHURi: DePARTMENT OF COMPUTER SCIENCE, MATHEMATICS AND STATISTICS, AMERICAN UNIVERSITY OF SHARJAH, P.O. BOX 26666, SHARJAH, UNITED ARAB EMIRATES

E-mail address: skhoury@aus.ac.ae 


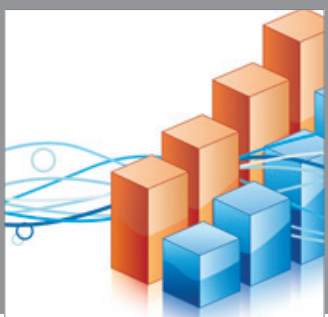

Advances in

Operations Research

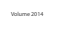

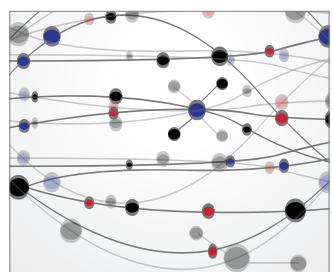

\section{The Scientific} World Journal
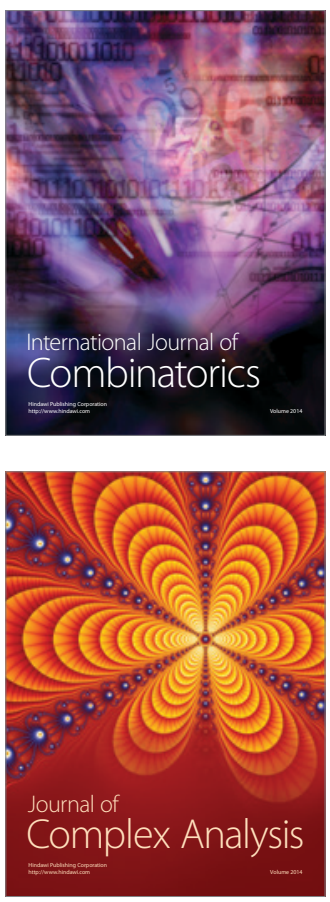

International Journal of

Mathematics and

Mathematical

Sciences
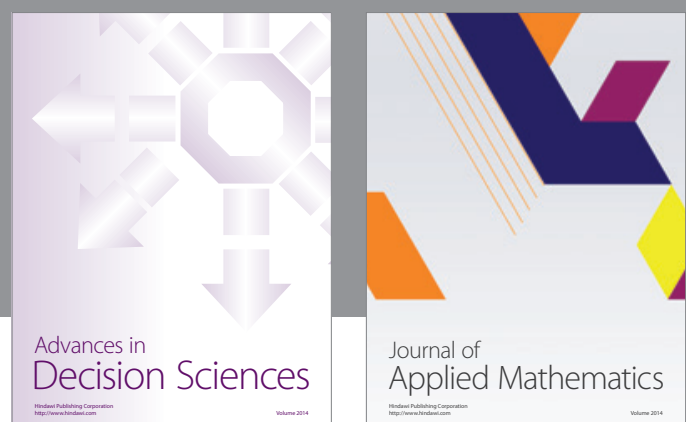

Journal of

Applied Mathematics
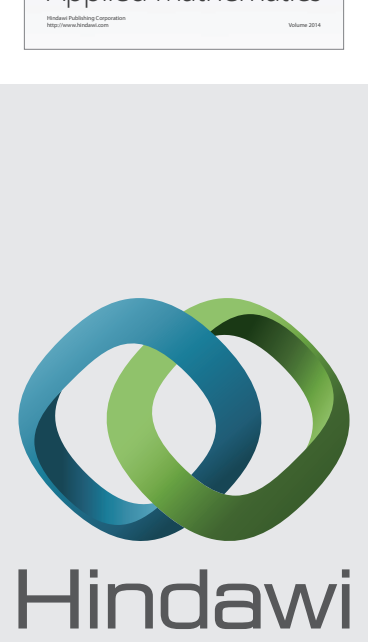

Submit your manuscripts at http://www.hindawi.com
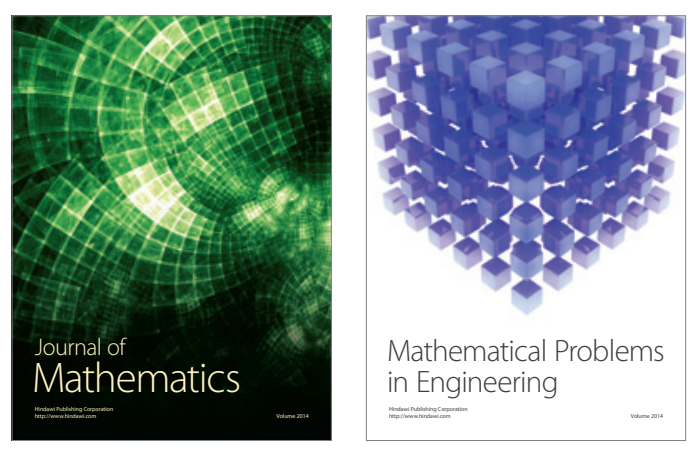

Mathematical Problems in Engineering
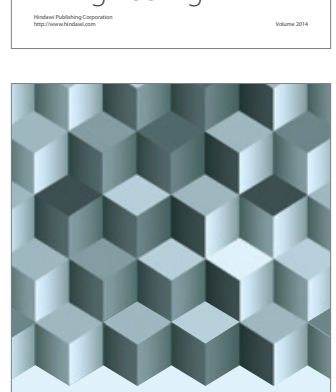

Journal of

Function Spaces
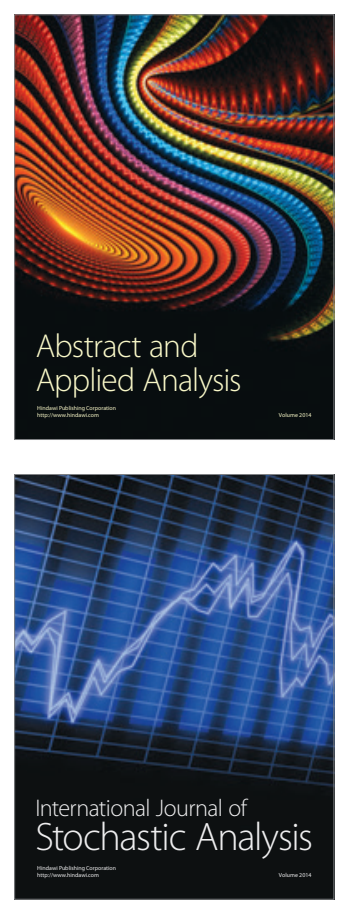

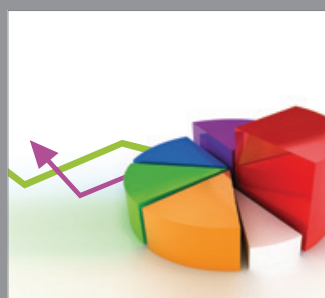

ournal of

Probability and Statistics

Promensencen
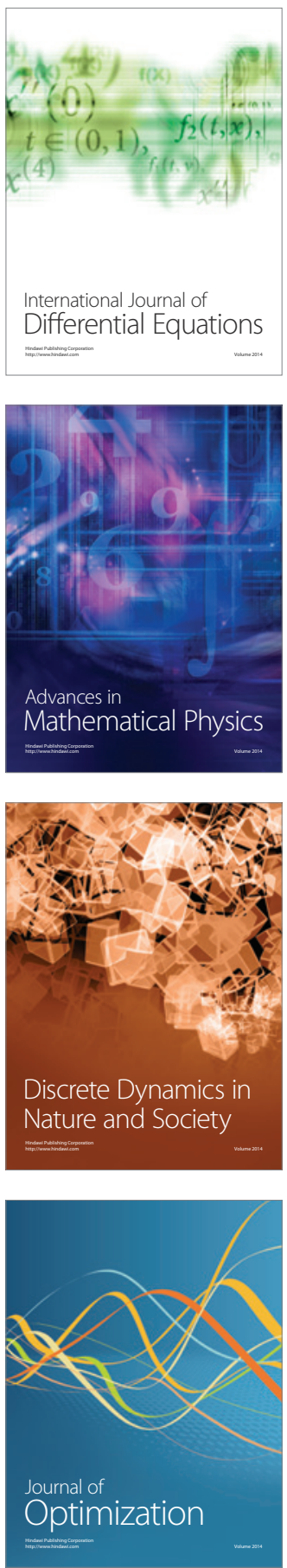